\title{
BMJ Open Effect of subsidies on healthful consumption: a protocol for a systematic review update
}

\author{
Fiona Pearson (D , ${ }^{1}$ Peijue Huangfu, ${ }^{2}$ Farah M Abu-Hijleh, ${ }^{3}$ Susanne F Awad, ${ }^{4}$ \\ Laith J Abu-Raddad (D) ,, Julia A Critchley ${ }^{2}$
}

To cite: Pearson $F$, Huangfu P, Abu-Hijleh FM, et al. Effect of subsidies on healthful consumption: a protocol for a systematic review update. BMJ Open 2020;10:e036031. doi:10.1136/ bmjopen-2019-036031

- Prepublication history and additional material for this paper are available online. To view these files, please visit the journal online (http://dx.doi. org/10.1136/bmjopen-2019036031).

Received 09 December 2019

Revised 29 May 2020

Accepted 02 July 2020

Check for updates

(c) Author(s) (or their employer(s)) 2020. Re-use permitted under CC BY-NC. No commercial re-use. See rights and permissions. Published by BMJ.

For numbered affiliations see end of article.

Correspondence to

Dr Fiona Pearson;

fiona.pearson2@ncl.ac.uk

\section{ABSTRACT}

Introduction The prevalence of diet-related noncommunicable diseases (NCDs) are rapidly increasing in most parts of the world. In order to ameliorate the related public health burden, evidence-informed policies to improve diet need to be implemented. Financial subsidies that promote healthful consumption patterns have the potential to reduce NCD risk and may also reduce inequality if targeted at those of low socio-economic position. This protocol is for an updated systematic review of such evidence.

Methods and analysis A systematic search strategy will be used to identify publications on fiscal intervention studies indexed in Embase, CINAHL, Web of Science, EconLit and PubMed in between January 2013 to February 2019. Two reviewers will independently sift identified citations using prespecified inclusion and exclusion criteria to inform full-text review. The outcomes of interest are: consumption patterns (\% change in targeted items and in overall dietary patterns), purchasing patterns (\% change) or body mass index. Pretested data capture forms will be used for double data extraction. Any inconsistencies in citation sifting or data extraction will be resolved by a third investigator and study authors will be contacted if needed. Systematic searches will be supplemented by reference checking of key articles. Study quality will be assessed and a narrative summary of findings will be produced. Meta-analyses and exploration of heterogeneity will be completed if appropriate.

Ethics and dissemination The review aims to strengthen findings of the primary studies it incorporates. It will synthesise existing published aggregated patient data and only present further aggregate data. Given this, no concerns are held relating to confidentiality and informed consent due to re-use of patient data.

If publications or data with ethical concerns are identified, they will be excluded from the review.

Results of the systematic review will be published in full and authors will engage directly with research audiences and key stakeholders to share findings.

PROSPERO registration number CRD42019125013

\section{INTRODUCTION}

Non-communicable diseases (NCDs) cause over 41 million deaths globally a year, this is approximately $71 \%$ of all global mortality. ${ }^{1}$ Of the deaths attributable to NCDs, 15 million

\section{Strengths and limitations of this study}

- This review will comprehensively summarise all identified quantitative data published on subsidies to promote healthful consumption in either a narrative, statistical summary or both.

- In order to optimise chances of identifying pertinent studies, a rigorous search strategy is being used and key papers identified will be searched for further relevant references.

- If plausible, an exploration of heterogeneity will be performed to help identify factors that may be influencing the efficacy of subsidies to promote healthful consumption.

- The strength of review findings will be highlighted using both a publication-centric and an outcomecentric evaluation of the evidence it includes.

- Although the review aims to be both rigorous and comprehensive, it may potentially be constrained by the primary data published on the topic. Previous findings will only be enhanced if sufficient new high quality data has been published.

are thought to be preventable, premature deaths occurring among those less than 75 years old. The majority of these preventable, premature deaths occur in low- and middleincome countries. In high-income countries NCDs disproportionately affect individuals from low socio-economic backgrounds. ${ }^{2}$ Noncommunicable diseases do not only impact the adult population. In 2018, an estimated 40 million children under-five were overweight increasing their risk of developing an NCD in later life. ${ }^{34}$ The potential future health burden due to NCDs is substantial.

Behavioural factors, including unhealthy diet, are thought to be responsible for much of the increase in prevalence of NCD risk factors such as overweight and obesity. ${ }^{5}$ Given diet is modifiable, promoting a healthy diet could have a large beneficial impact on the prevalence of NCD risk factors and lead to a reduction of the burden NCDs place on the healthcare system. To aid this, national and 
global governing health bodies have called for the implementation of evidence-based policies and transformation of the food system in order to make healthy diets accessible to everyone. ${ }^{6-9}$

A previous systematic review of data published up until 2014 identified that fiscal levers are effective in promoting healthful consumption patterns. ${ }^{1}$ Since 2014, published evidence has rapidly expanded and taxation policies have been implemented and scaled up at the local and national level. ${ }^{1-5}$ This planned systematic review will update a key component of the prior review, identifying a contemporary sample of publications that evaluate the effect of subsidies for food or beverages on the following outcomes of interest: consumption patterns ( $\%$ change in targeted items and \% change in overall dietary patterns), purchasing patterns (\% change in targeted items and \% change in non-targeted purchasing patterns) or body mass index (BMI, $\left.\mathrm{kg} / \mathrm{m}^{2}\right)$.

Prior review findings indicated that there might be differences in subsidy efficacy among people of different socio-economic status, but were underpowered to draw finite conclusions. ${ }^{6}$ Given this, the outlined review will also assess whether subsidy intervention effects are modified by intervention type or the following characteristics: age, sex, socio-economic status, country income level or ethnicity.

\section{METHODS AND ANALYSIS}

All data will be stored centrally and securely using institutional servers. The review process will be documented as standard and an annotated Preferred Reporting Items for Systematic Reviews and Meta-Analyses (PRISMA) flow diagram will be produced. The review findings will be reported in line with the PRISMA statement.

Search terms equivalent to those set out in the previous review of pricing interventions will be used to search MEDLINE, EconLit, Embase, CINAHL, Cochrane Library and Web of Science from January 2013 until February 2019 for published fiscal intervention studies. ${ }^{1}$

As an example, MEDLINE search terms that were used have been given below. Search terms for other databases can be found in online supplementary appendix 1 .

\footnotetext{
"National"[tiab] OR "Nationwide"[tiab] OR "state"[tiab] OR "statewide"[tiab] OR "city"[tiab] OR "Workplace" [MeSH Major Topic] OR "Workplace"[tiab] OR "Schools"[MeSH Terms] OR "School"[tiab] OR "School\$"[tiab] OR "Supermarket\$”[tiab] OR “restaurant\$”[tiab] OR "fast food" [tiab] OR "store\$" [tiab] OR "cafe"[tiab] OR "cafeteria" [tiab]

\section{AND}

"taxes" [MeSH Terms] OR "tax" [tiab] OR "taxation" [tiab] OR "subsidy" [tiab] OR "subsidies" [tiab] OR "incentive"[tiab] OR "price"[tiab] OR "pricing" [tiab] OR "voucher" [tiab] OR "coupon" [tiab] OR "rebate" [tiab] OR "elasticity"[tiab] OR "elasticities"[tiab]
}

AND

"Food and Beverages"[MeSH Terms] OR "fruit"[MeSH Terms] OR "fruit"[tiab] OR "vegetables"[MeSH Terms] OR "vegetables"[tiab] OR "fat" [tiab] OR "Sugar-sweetened beverage" [tiab] OR "soda" [tiab] OR "meat"[tiab] OR "dairy"[tiab] OR "candy" [tiab] OR "obesity" [MeSH Terms] OR "obesity"[tiab] OR "BMI" [tiab] OR "body weight" [MeSH Terms] OR "sodium, dietary" [MeSH Terms] OR "sodium"[tiab] OR "Body Mass Index"[MeSH Major Topic] OR "Adiposity"[MeSH Major Topic] OR "Adiposity" [tiab] OR "food consumption"[tiab] OR "Overweight" $[\mathrm{MeSH}]$ OR "Overweight"[tiab] OR "calorie"[tiab] OR "calorie\$”[tiab]

Where possible, affiliated search engines for online databases will be used to store electronic search strings and to download all identified citations into EndNote X7. Where no affiliated search engine exists, OvidSP will be used.

Participants will be generally healthy individuals (children or adults), and we will exclude any studies that focus only on those with specific illnesses (eg, diabetes mellitus, cardiovascular disease). We will not exclude interventions targeting those of lower socio-economic status.

Interventions of interest are subsidies that are being levied on the final product and that might prevent NCDs (eg, food or beverage price reduction via supermarket vouchers, or, incentive schemes in canteens to promote healthy eating). It is expected that most of the intervention studies in this field will not directly alter price but rather offer incentives (eg, vouchers) to increase purchases of healthier food such as fruits and vegetables. We will include studies of multicomponent interventions if the effect of the subsidy alone is discernible or the subsidy is the major component of the intervention. We acknowledge that some interventions might alter other food purchases (ie, those not being subsidised) and that some studies may lack reporting of results on overall food purchasing and/or consumption patterns, however, we will not exclude studies based on these characteristics. No exclusion will be made based on intervention setting or duration. We will include interventional and observational studies that assess the impact of subsidies on patterns of purchasing, consumption, participant's weight or BMI, as well as dietary quality and related outcomes. Studies will be excluded if they are animal studies, experimental studies or modelling studies. We will exclude interventions targeted at providers (eg; shops, schools, caterers) rather than consumers. We will exclude interventions aimed at snacks alone (eg, subsidies for vending machine use). We will not exclude publications based on language, year or status. However, studies will be excluded if price data is prior to 1990 , due to the potential temporality of food price variation and consumption.

Initial citation screening will be completed independently by two investigators in EndNote X7 using the outlined inclusion and exclusion criteria. As needed, 
publications will be retrieved in full and a prespecified, tested Excel form will be used to assess inclusion or exclusion of full-text publications. Where reviewers disagree on study inclusion or exclusion, a third independent reviewer will be consulted. References of key publications identified will be searched for further potential publications meeting inclusion criteria.

A prespecified, tested Excel form will also be used for data extraction including data on study quality and risk of bias. Data extraction will be completed independently by two investigators and any discrepancies in extraction resolved through consultation of a third independent reviewer. If at any point in the review process data is missing from a primary publication or is unclear study authors will be contacted for clarification.

To give a study-centric summary of quality the following validated tools will be used: the Newcastle Ottawa Score for observational studies, ${ }^{7}$ and Cochrane Risk of Bias 2 for randomised control trials. ${ }^{8}$ To highlight the consistency of evidence contributing to each outcome GRADE, a validated approach, will be used. ${ }^{9} 10$ GRADE incorporates its own outcome-centric assessment of quality.

\section{Strategy for data synthesis}

The characteristics of each study and intervention will be collated in a 'characteristics of included studies' table. If statistical synthesis of results is not appropriate, a structured report of the effects will be given. Quantitative data are likely to be available as aggregate level mean differences in purchasing, consumption, weight change (as defined by each individual intervention study) or BMI. If statistical pooling is feasible, a fixed or random effects Mantel-Haenszel models will be produced dependent on the heterogeneity present between studies (heterogeneity will be assessed using the $\chi^{2}$ test, the $\mathrm{I}^{2}$ statistic and the prediction interval). Given the broad clinical and methodological inclusion criteria it is likely that a random effects model will be used. Meta-analyses will be completed using Stata 15 with $\log$ files of all sessions stored. Alternate models will be compared in order to identify the model which best portrays the non-linear dose response relationships being explored.

Using subgroup analysis or meta-regression, as appropriate, potential causes of heterogeneity will be explored including; the site of intervention (eg, schools, workplaces, lower income communities, supermarkets), simultaneous delivery of additional intervention components (eg, education or taxation), mode of delivery (eg, online, face-to-face), level of subsidy (as a percentage change in price), compliance, adherence, duration of the study and its robustness to bias.

Sensitivity analyses will be completed to explore the impact on pooled intervention effect estimates of: study design, study size, study quality, study duration, study setting and of confounders adjusted for, data collection methods, withdrawals and drop-outs. Participant characteristics (eg, presence of comorbidities, average baseline weight), intervention features and different modes of delivery of the intervention (eg, through supermarkets, online vouchers or education) will also be noted and considered in sensitivity analyses.

Publication bias will be assessed using funnel plots if more than 10 studies are being included within a metaanalysis; Egger's test for bias will also be performed.

\section{Patient and public involvement}

This review has no element of patient or public involvement.

\section{Ethics and dissemination}

Given the nature of the systematic review process, ethical and safety considerations are minimal. No concerns are held relating to the re-use of patient data for new research or the need to seek further informed consent. The review will collate, synthesise and present previously published aggregated patient data. No concerns are held relating to patient confidentiality. There are no plans for data deposition or curation.

If publications or data with ethical insufficiency are identified by the review, although unlikely, they will be excluded.

Any amendments made to the protocol subsequent to its publication will initially be highlighted on PROSPERO, and indicated with a date. They will then be given as a dated addendum to this manuscript.

Findings will be published in a relevant academic journal and authors will engage with the wider field through academic fora and key stakeholders.

\section{Author affiliations}

${ }^{1}$ Evidence Synthesis Group, Population Health Sciences Institute, Newcastle University, Newcastle upon Tyne, UK

${ }^{2}$ Population Health Research Institute, St Georges's University of London, London, UK

${ }^{3}$ Department of Public Health, College of Health Sciences, Academic Quality Affairs Office, QU Health, Qatar University, Doha, Qatar

${ }^{4}$ Infectious Disease Epidemiology Group, Weill Cornell Medical College - Qatar, Cornell University, Qatar Foundation - Education City, Doha, Qatar

${ }^{5}$ Department of Healthcare Policy and Research, Weill Cornell Medicine, Cornell University, New York City, New York, USA

Contributors JAC and LAR conceptualised the study. FP designed the study protocol and drafted the manuscript. FP, JAC, LAR, PH, SK, SA and FA were involved in critically revising both the study protocol for this systematic review and the manuscript prepared from it. FP, JAC, LAR, PH, SK, SA and FA have approved the final version to be published and agreed to be accountable for all aspects of the work in ensuring that questions related to the accuracy or integrity of any part of the work are appropriately investigated and resolved.

Funding This publication was made possible by NPRP grant 10-1208-160017 from the Qatar National Research Fund (a member of Qatar Foundation).

Competing interests None declared.

Patient and public involvement Patients and/or the public were not involved in the design, or conduct, or reporting, or dissemination plans of this research.

Patient consent for publication Not required.

Provenance and peer review Not commissioned; externally peer reviewed.

Open access This is an open access article distributed in accordance with the Creative Commons Attribution Non Commercial (CC BY-NC 4.0) license, which permits others to distribute, remix, adapt, build upon this work non-commercially, and license their derivative works on different terms, provided the original work is 
properly cited, appropriate credit is given, any changes made indicated, and the use is non-commercial. See: http://creativecommons.org/licenses/by-nc/4.0/.

\section{ORCID iDs}

Fiona Pearson http://orcid.org/0000-0003-1626-0862

Laith J Abu-Raddad http://orcid.org/0000-0003-0790-0506

\section{REFERENCES}

1 Afshin A, Peñalvo JL, Del Gobbo L, et al. The prospective impact of food pricing on improving dietary consumption: a systematic review and meta-analysis. PLoS One 2017;12:e0172277.

2 Colchero MA, Rivera-Dommarco J, Popkin BM, et al. In Mexico, evidence of sustained consumer response two years after implementing a sugar-sweetened beverage Tax. Health Aff 2017;36:564-71.

3 Cawley J, Crain C, Frisvold D, et al. The Pass-Through of the largest tax on sugar-sweetened beverages: the case of Boulder. Colorado: National Bureau of Economic Research, 2018.

4 Cawley J, Frisvold D. The incidence of taxes on sugar-sweetened beverages: the case of Berkeley. California: National Bureau of Economic Research, 2015.
5 Cawley J, Frisvold D, Hill A, et al. The impact of the Philadelphia beverage tax on Purchases and consumption by adults and children. National Bureau of Economic Research, 2018.

6 Mizdrak A, Scarborough P, Waterlander WE, et al. Differential responses to food price changes by personal characteristic: a systematic review of experimental studies. PLoS One 2015;10:e0130320.

7 Wells G, Shea B, O'Connell D, et al. The Newcastle-Ottawa scale (NOS) for assessing the quality of nonrandomised studies in meta-analyses. Secondary the Newcastle-Ottawa scale (NOS) for assessing the quality of nonrandomised studies in meta-analyses. Available: http://www.ohri.ca/programs/clinical_epidemiology/oxford. asp

8 Sterne JAC, Savović J, Page MJ, et al. Rob 2: a revised tool for assessing risk of bias in randomised trials. $B M J ; 2: 14898$.

9 Brozek JL, Akl EA, Alonso-Coello P, et al. Grading quality of evidence and strength of recommendations in clinical practice guidelines. Part 1 of 3 . An overview of the grade approach and grading quality of evidence about interventions. Allergy 2009;64:669-77.

10 World Health Organization. Noncommunicable diseases country profiles 2018, 2018. 